\title{
Correction force for releasing crack tip element with XFEM and only discontinuous enrichment
}

\author{
How to smoothly release the crack tip element \\ with only discontinuous enrichment in XFEM?
}

\author{
Thomas Menouillard — Ted Belytschko \\ Theoretical and Applied Mechanics \\ Northwestern University, 2145 Sheridan Road \\ Evanston Illinois, 60208-3111, USA \\ \{t-menouillard, tedbelytschko\}@northwestern.edu
}

\begin{abstract}
This paper deals with numerical crack propagation and makes use of the extended finite element method in the case of explicit dynamics. The advantage of this method is the absence of remeshing. The use of XFEM with Heaviside functions only gives a binary description of the crack tip element: cut or not. Here, we modify the internal forces with a correction force in order to smoothly release the tip element while the virtual crack tip travels through an element. This avoids creating non physical stress waves and improves the accuracy of the evaluation of the stress intensity factors during propagation.

RÉSUMÉ. Cet article traite de la simulation numérique de propagation dynamique de fissure au moyen de la méthode des éléments finis étendus et d'une approche explicite. L'intérêt de cette méthode réside dans le non-remaillage. L'utilisation de la seule fonction de Heaviside dans la XFEM ne permettrait qu'une description binaire de l'élément de pointe de fissure : coupé, ou non coupé. Ici, on propose une correction des forces internes pour cet élément afin de le relaxer progressivement durant sa traversée par la fissure. Cela évite l'apparition d'ondes non physiques et améliore la précision du calcul des facteurs d'intensité des contraintes durant la propagation.

KEYWORDS: XFEM, cohesive law, discontinuous enrichment, Heaviside function, dynamic crack propagation.

MOTS-CLÉS : XFEM, loi cohésive, enrichissement discontinu, fonction Heaviside, propagation dynamique de fissure.
\end{abstract}

DOI:10.3166/EJCM.18.465-483 (c) 2009 Lavoisier, Paris 


\section{Introduction}

Classical finite elements are quite limited in dynamic crack propagation simulation because of the evolution of the topology of the domain and thus the use of a projection field on the updated discretization when the crack propagates. To adapt the standard finite element method to fracture computation, the extended finite element method (XFEM) has been developed, which completely avoids remeshing (Black et al., 1999; Moës et al., 1999). Also Belytschko et al. (2001) and Stolarska et al. (2001) combined this formulation with level sets. This XFEM method is based on the partition of unity pioneered by Melenk et al. (1996), whereby specific functions are used to describe the physical behavior in subdomains of the problem. Thus in (Moës et al., 1999; Moës et al., 2002) the discontinuous enrichment function was used along the crack in order to describe a discontinuous displacement. Réthoré et al. (2005) and Combescure et al. (2008) ensure the energy conservation during the propagation. Dolbow et al. (2001) and Khenous et al. (2006) dealt with contact in the XFEM formulation.

The well known explicit central difference method (Belytschko et al., 2000) is usually used for dynamic crack propagation. For the XFEM formulation, Menouillard et al. (2006b) developed a mass lumping strategy for the discontinuous enrichment part. They found that the enrichment does not decrease the stability time step much; indeed the XFEM critical time step is at least $70 \%$ of the finite element one. Then, Menouillard et al. (2008) used another decomposition of the enriched shape function developed by Hansbo et al. (2004) which is used in the phantom node method developed by Song et al. (2006), and extended for 3D by Duan et al. (2009). Menouillard et al. (2009) have presented an enriched formulation, including a moving crack tip enrichment to enhance the crack tip description. Substantial improvement was observed in the results, but this formulation is more complex.

In this paper, we describe a method to deal with the description of the crack tip element in term of stiffness relaxation without using any tip enrichment function. It aims at characterizing the release of the crack tip element when the crack propagates through. An additional correction force will be introduced in this crack tip element, which leads the new additional degrees of freedom release continuously. Whereas no tip enrichment is used to describe the crack tip vicinity, the releasing process reduces spurious oscillatory stress waves.

The paper is organized as follows. Section 2 presents the governing equation of the problem, the cohesive zone model used and the stress intensity factors computation. Section 3 describes the space discretization of the XFEM formulation using only the discontinuous enrichment. Section 4 describes the new method for releasing the crack tip element by using correction force: it explains the relation between the correction force and the position of the virtual crack tip in the element. Section 5 presents two numerical examples to underline the effect of the correction force in the stress field in the structure and in the computation of the stress intensity factors. 


\section{General problem modeling}

Consider an initial domain $\Omega^{0}$ and its boundary $\Gamma^{0}$ as shown in Figure 1: $\partial \Omega_{u}^{0} \cup$ $\partial \Omega_{F}^{0}=\Gamma^{0}$ and $\partial \Omega_{u}^{0} \cap \partial \Omega_{F}^{0}=\emptyset$. In the current domain, the image of $\Omega^{0}$ is denoted $\Omega$, and the motion is described by $\mathbf{x}=\Phi(\mathbf{X}, t)$, where $t$ is the time, and $\mathbf{X}$ and $\mathbf{x}$ the material and spatial coordinates, respectively. The displacement at the material point $\mathbf{X}$ is denoted by $\mathbf{u}(\mathbf{X}, t)$. Figure 1 describes the reference and the current domains, and the different boundaries and their corresponding conditions.

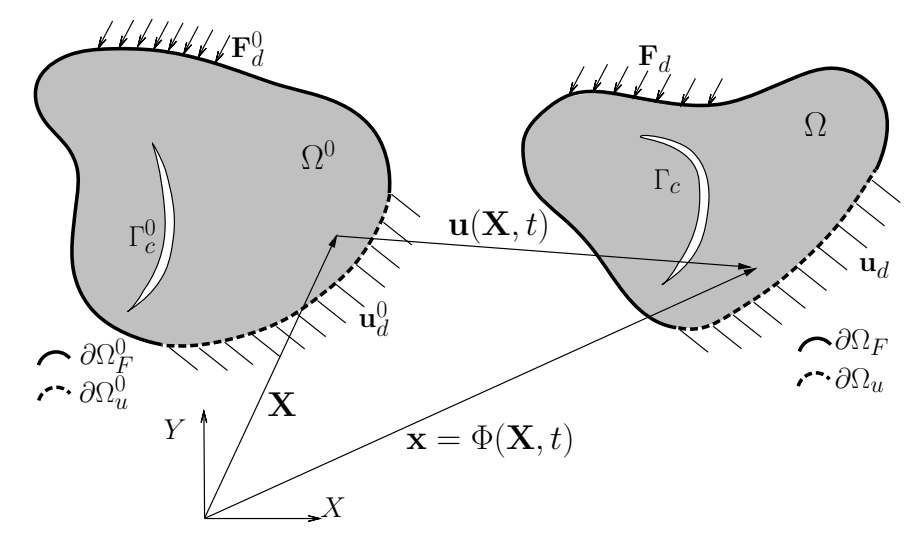

Figure 1. A two dimensional body with a discontinuity and its representation in the reference and the current domains

\subsection{Updated lagrangian formulation}

The momentum equation in the updated Lagrangian form is written as:

$$
\rho \ddot{\mathbf{u}}=\operatorname{div}(\underline{\underline{\sigma}})+\mathbf{f}_{d} \quad \text { in } \Omega
$$

where $\rho$ is the density, and $\mathbf{f}_{d}$ a body force vector on the current domain $\Omega, \underline{\sigma}$ the Cauchy stress tensor, and $\ddot{\mathbf{u}}$ the acceleration. The global equation verified by the displacement solution $\mathbf{u} \in \mathcal{U}$ is (see e.g. Belytschko et al. (2000)):

$$
\begin{gathered}
\int_{\Omega / \Gamma_{c}} \quad \rho \ddot{\mathbf{u}} \cdot \mathbf{v} d \Omega+\int_{\Omega / \Gamma_{c}} \underline{\underline{\sigma}}: \underline{\underline{\varepsilon}}(\mathbf{v}) d \Omega=\int_{\Omega / \Gamma_{c}} \mathbf{f}_{d} \cdot \mathbf{v} d \Omega \\
+\int_{\partial \Omega_{F}} \mathbf{F}_{d} \cdot \mathbf{v} d \Gamma+\int_{\Gamma_{c}} \mathbf{F}_{c o h} \cdot \mathbf{v} d \Gamma
\end{gathered}
$$

where $\underline{\underline{\varepsilon}}$ is the strain tensor, and $\mathbf{v} \in \mathcal{U}_{0}$ :

$$
\mathcal{U}=\left\{\mathbf{u} \mid \mathbf{u}(\mathbf{x})=\mathbf{u}_{d}, \forall \mathbf{x} \in \partial \Omega_{u}, \text { and regularity of } \mathbf{u}\right\}
$$




$$
\mathcal{U}_{0}=\left\{\mathbf{u} \mid \mathbf{u}(\mathbf{x})=\mathbf{0}, \forall \mathbf{x} \in \partial \Omega_{u}, \text { and regularity of } \mathbf{u}\right\}
$$

The boundary of the current domain $\Omega$ is partitioned into $\partial \Omega_{u}$ on which displacements $\mathbf{u}_{d}$ are prescribed, $\partial \Omega_{F}$ on which tractions $\mathbf{F}_{d}$ are prescribed, and then $\Gamma_{c}$ which corresponds to the displacement discontinuity, i.e. crack. One can sum up by writing:

$$
\left\{\begin{aligned}
\mathbf{u}(\mathbf{x}, t) & =\mathbf{u}_{d} & \forall \mathbf{x} \in \partial \Omega_{u}, \forall t \text { in }[1, T] \\
\underline{\underline{\sigma}}(\mathbf{x}, t) \cdot \mathbf{n} & =\mathbf{F}_{d} & \forall \mathbf{x} \in \partial \Omega_{F}, \forall t \text { in }[1, T] \\
\underline{\underline{\sigma}}(\mathbf{x}, t) \cdot \mathbf{n} & =\mathbf{F}_{c o h} & \forall \mathbf{x} \in \Gamma_{c}, \forall t \text { in }[1, T]
\end{aligned}\right.
$$

where $\mathbf{n}$ denotes outer normal of the boundary $\partial \Omega, T$ is the total time. Moreover, the latter boundary $\Gamma_{c}$ also depends on time as the crack propagates: $\Gamma_{c}(t)$. Note that $\partial \Omega=\partial \Omega_{u} \cup \partial \Omega_{F} \cup \Gamma_{c}$ and $\partial \Omega_{u} \cap \partial \Omega_{F}=\emptyset, \partial \Omega_{u} \cap \Gamma_{c}=\emptyset, \partial \Omega_{F} \cap \Gamma_{c}=\emptyset$.

\subsection{Total lagrangian formulation}

The momentum equation in the total Lagrangian form is written as:

$$
\rho_{0} \ddot{\mathbf{u}}=\operatorname{div}(\underline{\underline{P}})+\mathbf{f}_{d}^{0} \quad \text { in } \Omega^{0}
$$

where $\rho_{0}$ is the initial density, and $\mathbf{f}_{d}^{0}$ a body force vector on the initial domain $\Omega^{0}, \underline{\underline{P}}$ the nominal stress tensor, and $\ddot{\mathbf{u}}$ the acceleration. The weak form of the momentum equation is:

$$
\begin{array}{cc}
\int_{\Omega^{0} / \Gamma_{c}^{0}} \quad \rho_{0} \ddot{\mathbf{u}} \cdot \mathbf{v} d \Omega^{0}+\int_{\Omega^{0} / \Gamma_{c}^{0}} \underline{\underline{P}}: \underline{\underline{F}}(\mathbf{v}) d \Omega^{0}=\int_{\Omega^{0} / \Gamma_{c}^{0}} \mathbf{f}_{d} \cdot \mathbf{v} d \Omega^{0} \\
+\int_{\partial \Omega_{F}^{0}} \mathbf{F}_{d}^{0} \cdot \mathbf{v} d \Gamma^{0}+\int_{\Gamma_{c}^{0}} \mathbf{F}_{c o h}^{0} \cdot \mathbf{v} d \Gamma^{0}
\end{array}
$$

where $\underline{\underline{F}}$ is the deformation gradient, and $\mathbf{v} \in \mathcal{T}_{0}$ :

$$
\begin{aligned}
& \mathcal{T}=\left\{\mathbf{u} \mid \mathbf{u}(\mathbf{x})=\mathbf{u}_{d}^{0}, \forall \mathbf{x} \in \partial \Omega_{u}^{0}, \text { and regularity of } \mathbf{u}\right\} \\
& \mathcal{T}_{0}=\left\{\mathbf{u} \mid \mathbf{u}(\mathbf{x})=\mathbf{0}, \forall \mathbf{x} \in \partial \Omega_{u}^{0}, \text { and regularity of } \mathbf{u}\right\}
\end{aligned}
$$

The boundary of the initial domain $\Omega^{0}$ is partitioned into $\partial \Omega_{u}^{0}$ on which displacements $\mathbf{u}_{d}^{0}$ are prescribed, $\partial \Omega_{F}^{0}$ on which tractions $\mathbf{F}_{d}^{0}$ are prescribed, and then $\Gamma_{c}^{0}$ which corresponds to the displacement discontinuity, i.e. crack in the initial domain. The boundary conditions are summarized as

$$
\left\{\begin{array}{rlrl}
\mathbf{u}(\mathbf{x}, t) & =\mathbf{u}_{d}^{0} & & \forall \mathbf{x} \in \partial \Omega_{u}^{0}, \forall t \in[1, T] \\
\underline{P}(\mathbf{x}, t) \cdot \mathbf{n}^{0} & =\mathbf{F}_{d}^{0} & \forall \mathbf{x} \in \partial \Omega_{F}^{0}, \forall t \in[1, T] \\
\underline{\underline{P}}(\mathbf{x}, t) \cdot \mathbf{n}^{0} & =\mathbf{F}_{c o h}^{0} & \forall \mathbf{x} \in \Gamma_{c}^{0}, \forall t \in[1, T]
\end{array}\right.
$$


where $\mathbf{n}^{0}$ denotes outer normal of the boundary $\partial \Omega^{0}, T$ is the total time. Moreover, the latter boundary $\Gamma_{c}^{0}$ also depends on time as the crack propagates: $\Gamma_{c}^{0}(t)$. Note that $\partial \Omega^{0}=\partial \Omega_{u}^{0} \cup \partial \Omega_{F}^{0} \cup \Gamma_{c}^{0}$ and $\partial \Omega_{u}^{0} \cap \partial \Omega_{F}^{0}=\emptyset, \partial \Omega_{u}^{0} \cap \Gamma_{c}^{0}=\emptyset, \partial \Omega_{F}^{0} \cap \Gamma_{c}^{0}=\emptyset$.

\subsection{Cohesive law}

An important issue when considering failure is the observation that most engineering materials are not perfectly brittle in the Griffith theory, but become more ductile after reaching the strength limit. This fracture process zone is located in front of the crack tip, and its size characterizes a linear-elastic fracture, or a ductile one. The cohesive forces that exist in this fracture zone needs to be taken into account. A good way is to use cohesive-zone models, which were introduced first by Barenblatt (1962) for elastic plastic fracture in ductile metals, and by Hillerborg et al. (1976) for brittle materials. Several works recently focused on the cohesive crack law (Benvenuti, 2008; Unger et al., 2007; Patzák et al., 2003). Figure 2 shows the crack opening and the tensile stress linked with the position along the crack. It underlines the closing effect near the crack tip with a limit opening $\delta_{\max }$; above this opening, there is no more tensile stress, because the crack after this point is completely open.

Figure 3 presents a linear cohesive law (Ortiz et al., 1999; Remmers et al., 2003; Rabczuk et al., 2009); the area under the curve represents the fracture energy $G_{F}$, defined by:

$$
G_{F}=\int_{0}^{\delta_{\max }} \tau(\delta) d \delta=\frac{1}{2} \tau_{\max } \delta_{\max }
$$

where $\tau$ denotes the tensile stress along the crack, and $\tau_{\max }, \delta_{\max }$ define the linear cohesive law described in Figure 3.

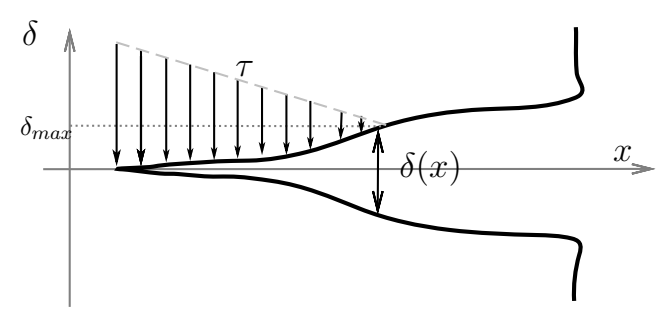

Figure 2. Cohesive law described with the crack opening $\delta$ as a function of the position $x$ along the crack

\subsection{Stress intensity factors}

The main step consists in relating the propagation of the crack to the state of the structure, in order to evaluate the direction and velocity of the crack as functions of 
time, denoted respectively by $\theta_{c}(t)$ and $\dot{a}(t)$. The initial value $a(0)=a_{0}$ is the initial crack length, and the knowledge of the two functions $a(t)$ and $\theta_{c}(t)$ are sufficient to describe the whole history of the crack geometry in the structure. As we assume that the material is homogeneous with linear isotropic behavior, the problem falls within the framework of the linear dynamic fracture mechanics in which the energy release rate denoted by $\mathcal{G}$, can be defined. The dynamic stress intensity factors $K_{1}$ and $K_{2}$ are defined by the asymptotic behavior of the stress near crack tip as (Bui, 1978) (local coordinates system near crack tip):

$$
\begin{aligned}
& K_{1}=\lim _{r \rightarrow 0} \sqrt{2 \pi r} \sigma_{y y} \\
& K_{2}=\lim _{r \rightarrow 0} \sqrt{2 \pi r} \sigma_{x y}
\end{aligned}
$$

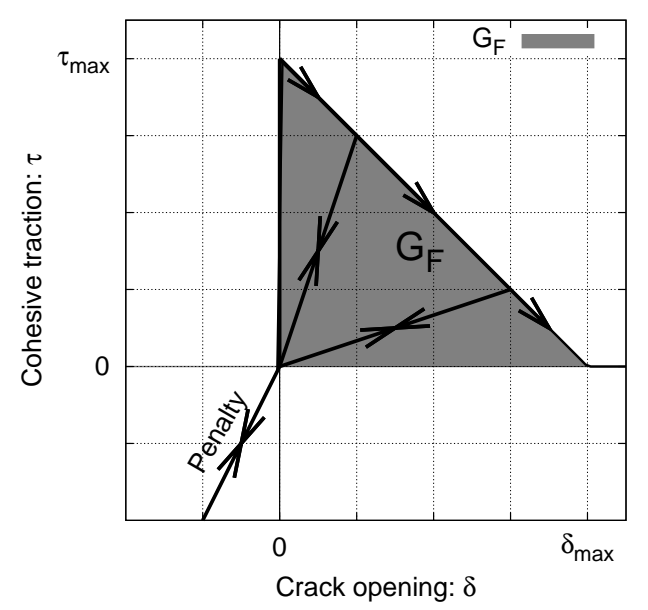

Figure 3. Linear cohesive law: the area in gry under the curve represents the fracture energy $G_{F}$

The relation between the energy release rate and the stress intensity factors is given by Freund (Freund, 1990) and Bui (Bui, 1978) as:

$$
\mathcal{G}=\frac{1-\nu^{2}}{E}\left(\beta_{1}(\dot{a}) K_{1}^{2}+\beta_{2}(\dot{a}) K_{2}^{2}\right)
$$


where $E$ denotes the Young's modulus, $\nu$ the Poisson's ratio, $\dot{a}$ the crack velocity, and $\beta_{i}$ are the universal functions defined by:

$$
\begin{aligned}
\beta_{i}(\dot{a}) & =\frac{4 \alpha_{i}\left(1-\alpha_{2}^{2}\right)}{(\kappa+1) D(\dot{a})} \quad i \in\{1,2\} \\
\alpha_{i} & =\sqrt{1-\left(\frac{\dot{a}}{c_{i}}\right)^{2}} \quad i \in\{1,2\} \\
D(\dot{a}) & =4 \alpha_{1} \alpha_{2}-\left(1+\alpha_{2}^{2}\right)^{2}
\end{aligned}
$$

$c_{1}$ and $c_{2}$ are respectively the dilatational and shear velocities, and are given as a function of the Lamé coefficients and the density as $c_{1}=\sqrt{\frac{\lambda+2 \mu}{\rho}}$ and $c_{2}=\sqrt{\frac{\mu}{\rho}}$. The Kolosov coefficient $\kappa$ is $3-4 \nu$ for the plane strain, and $(3-\nu) /(1+\nu)$ for the plane stress. $D$ is a function whose zero defines the Rayleigh wave celerity denoted by $c_{r}$. The dynamic energy release rate predicts a propagation by comparing its value to the critical one $\mathcal{G}_{c}$, whereas the knowledge of the stress intensity factors inform about the direction of the propagation. The knowledge of the two stress intensity factors $K_{1}$ and $K_{2}$ is necessary to evaluate the importance of each mode (Bui, 1978), and so the new crack direction. The computation of the different stress intensity factors is based on the auxiliary fields near the crack tip and the interaction integral which allows to write two scalar equations, and determine the two unknown stress intensity factors $K_{1}$ and $K_{2}$. Further developments of the interaction integral are presented by Freund (1990), Réthoré et al. (2005).

The stress intensity factors $K_{1}$ and $K_{2}$ are computed using a domain independent integral $I^{\text {int }}$ and a virtual crack extension $\mathbf{q}$ by:

$$
\begin{aligned}
& I^{i n t}=-\int_{\Omega}\left(\underline{\underline{\sigma}}^{\text {aux }}: \underline{\underline{\nabla}}^{\mathbf{u}}-\rho \dot{\mathbf{u}}^{a u x}\right) \operatorname{div}(\mathbf{q}) d \Omega \\
& +\int_{\Omega} \underline{\underline{\sigma}}^{a u x}:(\underline{\underline{\nabla}} \mathbf{u} \underline{\underline{\nabla}} \mathbf{q})+\underline{\underline{\sigma}}:\left(\underline{\underline{\nabla}} \mathbf{u}^{a u x} \underline{\underline{\nabla}} \mathbf{q}\right) d \Omega \\
& +\int_{\Omega} \operatorname{div}\left(\underline{\underline{\sigma}}^{a u x}\right) \underline{\underline{\nabla}} \mathbf{u}(\mathbf{q})+\rho \ddot{\mathbf{u}} \underline{\underline{\nabla}} \mathbf{u}^{a u x} d \Omega \\
& +\int_{\Omega} \rho \dot{\mathbf{u}}^{a u x} \underline{\underline{\nabla}} \dot{\mathbf{u}}(\mathbf{q})+\rho \dot{\mathbf{u}} \underline{\underline{\mathbf{u}}}^{\text {aux }}(\mathbf{q}) d \Omega
\end{aligned}
$$

where $\underline{\underline{\sigma}}^{a u x}, \mathbf{u}^{\text {aux }}$ are auxiliary stress and displacement fields. The vector $\mathbf{q}$, parallel to the crack, is defined by:

$$
\mathbf{q}= \begin{cases}\mathbf{0} & \text { outside the surface } S_{1} \cup S_{2} \\ \|\mathbf{q}\|=1 & \text { inside the surface } S_{1} \\ \|\mathbf{q}\| \text { linear } & \text { inside the surface } S_{2}\end{cases}
$$


where $S_{1}$ and $S_{2}$ are surfaces defined near crack tip. Figure 4 shows the surfaces $S_{1}$ and $S_{2}$, and presents the direction and the norm of the virtual extension field q near crack tip. Note that the shape of the surfaces $S_{1}$ and $S_{2}$ can be circular too. Then this integral is:

$$
I^{\text {int }}=\frac{2\left(1-\nu^{2}\right)}{E}\left(\beta_{1}(\dot{a}) K_{1} K_{1}^{\text {aux }}+\beta_{2}(\dot{a}) K_{2} K_{2}^{\text {aux }}\right)
$$

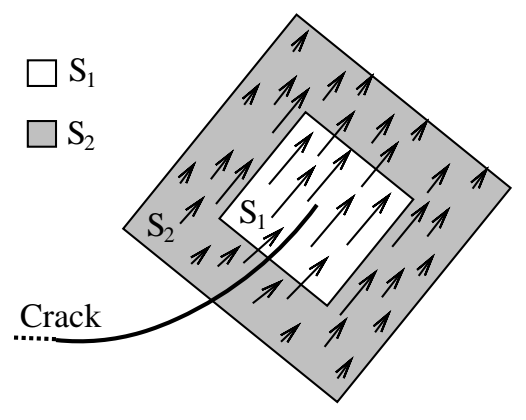

Figure 4. Direction and norm of the virtual extension field $\mathbf{q}$

The different stress intensity factors are estimated through an appropriate choice of $\mathbf{u}^{a u x}$ : i.e. $K_{1}^{a u x}=1$ and $K_{2}^{a u x}=0$ for the determination of $K_{1}$, and $K_{1}^{a u x}=0$ and $K_{2}^{a u x}=1$ for the determination of $K_{2}$. Two equations are needed to compute the two stress intensity factors (Menouillard et al., 2006a).

The equivalent dynamic stress intensity factor $K_{\theta \theta}$ is defined by:

$$
K_{\theta \theta}=\cos ^{3}\left(\frac{\theta_{c}}{2}\right) K_{1}-\frac{3}{2} \cos \left(\frac{\theta_{c}}{2}\right) \sin \theta_{c} K_{2}
$$

Freund (1972) develops a relation between the dynamic energy release rate and the crack velocity. Freund et al. (1982) and Rosakis et al. (1982) have linked experimentally, the crack velocity to the stress intensity factors through the relation explained by Freund (1972):

$$
\dot{a}=\left\{\begin{array}{cc}
0 & \text { if } K_{\theta \theta}<K_{1 c} \\
c_{r}\left(1-\left(\frac{K_{1 c}}{K_{\theta \theta}}\right)^{2}\right) & \text { otherwise }
\end{array}\right.
$$

In addition, the maximum hoop stress criterion drives the direction of the crack. So a crack increment in the explicit algorithm is defined by $\Delta a=\dot{a} \Delta t$, where $\Delta t$ is the time step size. 


\section{Space discretization and kinematics}

In XFEM, the enrichments are added to the conventional finite element approximation according to the partition of unity method. The function, denoted by $H$ in this paper, takes into account the physical phenomenon. This discontinuous function $H$ is typically used to describe a displacement discontinuity across the crack; its value, through the level set $f$, is +1 on one side of the crack and -1 on the other (see (Belytschko et al., 2001)). Thus, the discretized displacement $\tilde{\mathbf{u}}$ is written using an enriched basis as:

$$
\tilde{\mathbf{u}}(\mathbf{x}, t)=\sum_{I \in \mathcal{N}} N_{I}(\mathbf{x}) \mathbf{u}_{I}+\sum_{J \in \mathcal{N}_{c u t}} H(f(\mathbf{x})) N_{J}(\mathbf{x}) \mathbf{b}_{J}
$$

where $\mathcal{N}$ is the set of all nodes of the mesh, $\mathcal{N}_{\text {cut }}$ the set of nodes belonging to the elements completely cut by the crack. The standard shape functions are denoted by $N_{I}$, and $H$ denotes the discontinuous Heaviside function. The standard degrees of freedom are denoted by $\mathbf{u}_{I} ; \mathbf{b}_{J}$ corresponds to the enriched degrees of freedom associated with the function $H$.

\section{Correction force: releasing crack tip element}

In this section we aim at developing a method intended to deal with the release of the crack tip element when the crack propagates through it. It is done in order to avoid sudden element relaxation near the crack tip during propagation, and thus the creation of non physical stress waves.

Figure 5a shows the crack tip element with the associated phantom nodes. The discrete momentum equation associated is:

$$
\mathbb{M} \cdot \ddot{\mathbf{U}}=\mathbf{f}^{\text {ext }}-\mathbf{f}^{\text {int }}+\mathbf{f}^{\text {coh }}
$$

where $\mathbb{M}$ is the mass matrix, $\ddot{\mathbf{U}}$ the nodal acceleration, $\mathbf{f}^{\text {int }}$ the internal force, $\mathbf{f}^{\text {ext }}$ the external force, and $\mathbf{f}^{\text {coh }}$ the cohesive force. When the crack tip reaches the next element, the new crack tip element is suddenly released (see the sudden passage from Figure 5a to 5d); even with the cohesive force the corresponding internal force takes a significant value when the phantom nodes are injected (i.e. $\mathbf{f}_{\mathbf{a}}^{\text {int }}$ is non zero in Figure $5 d)$.

Our proposed method makes a progressive release of the crack tip element. Thus, Figures $5 \mathrm{~b}$ and $5 \mathrm{c}$ show the crack tip element on a dotted line and the additional correction force acting in the momentum equation, which aims at relaxing smoothly the element when the crack tip travels through from one edge of the element to the next one. Thus the modified discrete momentum equation becomes

$$
\mathbb{M} \cdot \ddot{\mathbf{U}}=\mathbf{f}^{\text {ext }}-\mathbf{f}^{\text {int }}+\mathbf{f}^{\text {coh }}+\mathbf{f}^{\text {correct }}
$$

where $\left\|\mathbf{f}^{\text {correct }}\right\|$ tends to zero when the crack tip reaches the new edge, and thus the element becomes completely cut by the discontinuity (see Figure 5d). 


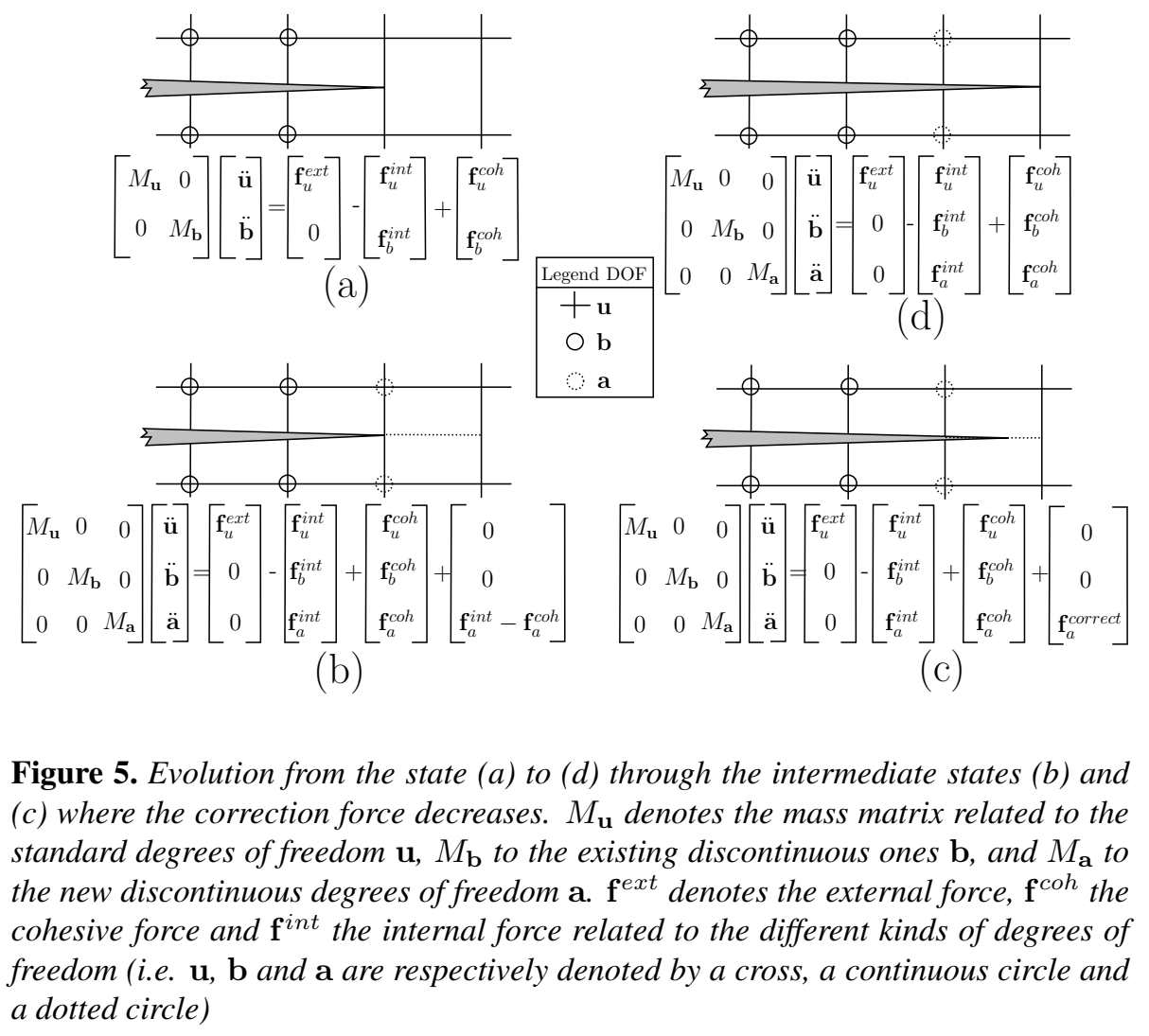

The initial value of the correction force (when the crack tip is on the previous edge) is such that the sum of the four forces in the equation above is zero as shown in Figure 5b. At this point, the correction force is the same than the internal force, and thus no acceleration occurs yet on the new additional degrees of freedom, denoted by $\mathbf{a}$ in Figure 5. The evolution of the correction force is shown in Figure 6 which describes the magnitude of the correction force as a function of the crack tip position in the tip element. Indeed the correction force goes from the initial internal force to zero when the crack tip travels from one edge to the next one. Between these two crack tip positions, the correction force is taken to be linear in our method. However there is no restriction to use a linear law. The continuity of the internal force related to the new additional degrees of freedom, gives the same property to the acceleration through the momentum equation. Therefore, the velocity and displacement remains quite continuous in time when additional degrees of freedom are injected, and thus the property of continuity in time remains in the strain and stress field too, and a continuous progressive release of the tip element occurs. 


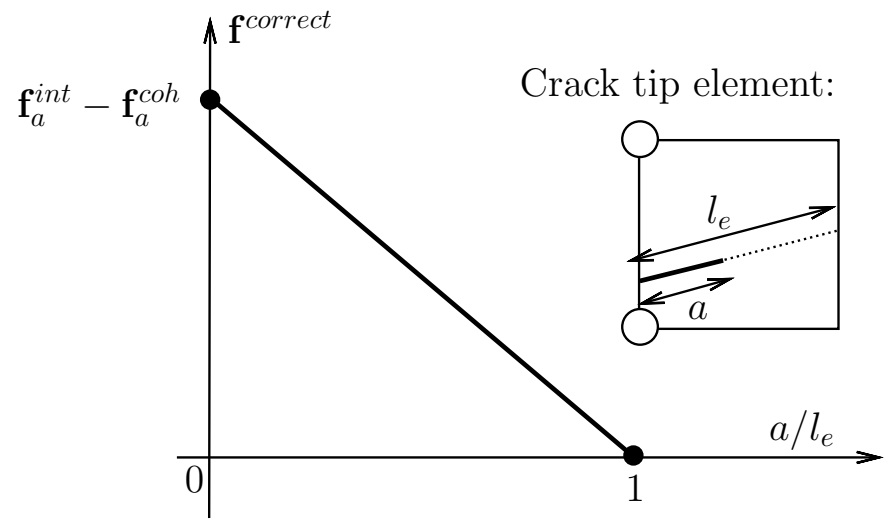

Figure 6. Law of the correction force as a function of the fracture ratio of the crack tip element (i.e. the position of the crack tip in the element)

\section{Numerical applications}

Two numerical examples are illustrated: the first one aims at showing the improvement in term of accuracy in stress intensity factors computation, and the second one verifies that the correction force does not modify the energy conservation during computation.

\subsection{Moving semi-infinite mode 1 crack}

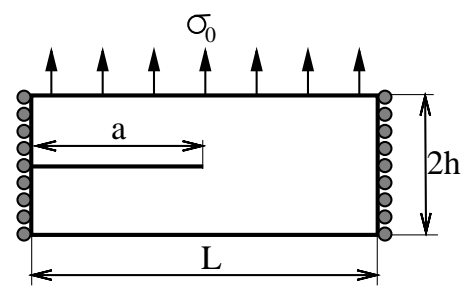

Figure 7. Geometry and loading of the semi-infinite plate example

The example considered in this paragraph is an infinite plate with a semi-infinite crack (Ravi-Chandar, 2004) loaded as shown in Figure 7. A theoretical solution of this problem for a given crack velocity is given in Freund (Freund, 1990). Since this analytical solution was obtained under the assumptions of infinite plate with a semiinfinite crack and a given speed of the crack's tip, according to the geometry described in Figure 7, those could be compared for time $t \leq 3 t_{c}=3 h / c_{1}$ (where $c_{1}$ is the 
dilatational wave speed). Beyond that, the reflected stress wave reaches the crack's tip and the analytical solution is no longer valid. The dimensions of the structure are the following: the length is $L=10 \mathrm{~m}$, the initial crack length $a=5 \mathrm{~m}$, and the vertical position of the crack is $h=2 \mathrm{~m}$. Two regular meshes are used: 78x39 and 120x59 4-node elements. The material properties of the linear elastic media are given in Table 1 . The tensile stress applied on the top surface is $\sigma_{0}=500 \mathrm{MPa}$. The crack velocity is imposed to be zero until $1.5 t_{c}$, and $1,500 \mathrm{~m} / \mathrm{s}$ after. The mode 1 stress intensity factor is normalized by the factor $\sigma_{0} \sqrt{h}$.

Table 1. Material properties for the moving crack experiment in pure mode 1

\begin{tabular}{lcr}
\hline Material property & Symbol & Value \\
\hline Young's modulus & $E$ & $210 \mathrm{GPa}$ \\
Poisson's ratio & $\nu$ & 0.3 \\
Density & $\rho$ & $8,000 \mathrm{~kg} / \mathrm{m}^{3}$ \\
\hline
\end{tabular}

Let us study the effect of a moving crack on the accuracy of the stress intensity factor using or not the correction force described previously. So, one has the analytical relation between the stress intensity factor $K_{1}$ and the velocity $\dot{a}$ of the crack (Freund, 1990):

$$
K_{1}(\dot{a}, t)= \begin{cases}0 & \text { if } t<t_{c} \\ \frac{2 \sigma_{0}}{1-\nu} \sqrt{\frac{c_{1}\left(t-t_{c}\right)(1-2 \nu)}{\pi}} & \text { if } t_{c} \leq t<1.5 t_{c} \\ \frac{2 \sigma_{0}}{1-\nu} \sqrt{\frac{c_{1}\left(t-t_{c}\right)(1-2 \nu)}{\pi}} \frac{1-\frac{\dot{a}}{c_{r}}}{1-\frac{a_{2}}{2 c_{r}}} & \text { if } 1.5 t_{c} \leq t\end{cases}
$$

where the Rayleigh wave speed is $c_{r}=2,947 \mathrm{~m} / \mathrm{s}$ and the dilatational wave speed is $c_{1}=5,944 \mathrm{~m} / \mathrm{s}$.

Figure 8, respectively 10, presents the normalized stress intensity factor as a function of time for the coarse mesh, respectively fine mesh, with and without the correction force. Both figures underline that the correction force improves the result during propagation by decreasing the magnitude of the oscillations due to the released crack tip element. Indeed the number of oscillations are directly related to the number of newly cracked elements. To evaluate the improvement, Figures 9 and 11 show the relative error between the computations using the correction force and not for the coarse and fine meshes. The error is decreased from $20 \%$ to $5 \%$ just by adding the correction force. Figure 12 shows the stress state in the structure at the end of the computation, for the two cases: with and without the correction force. One can notice the correction force makes the stress smoother in the structure when the crack propagation occurs, and erases all the release stress waves due to the injected phantom node as shown in the upper part. 


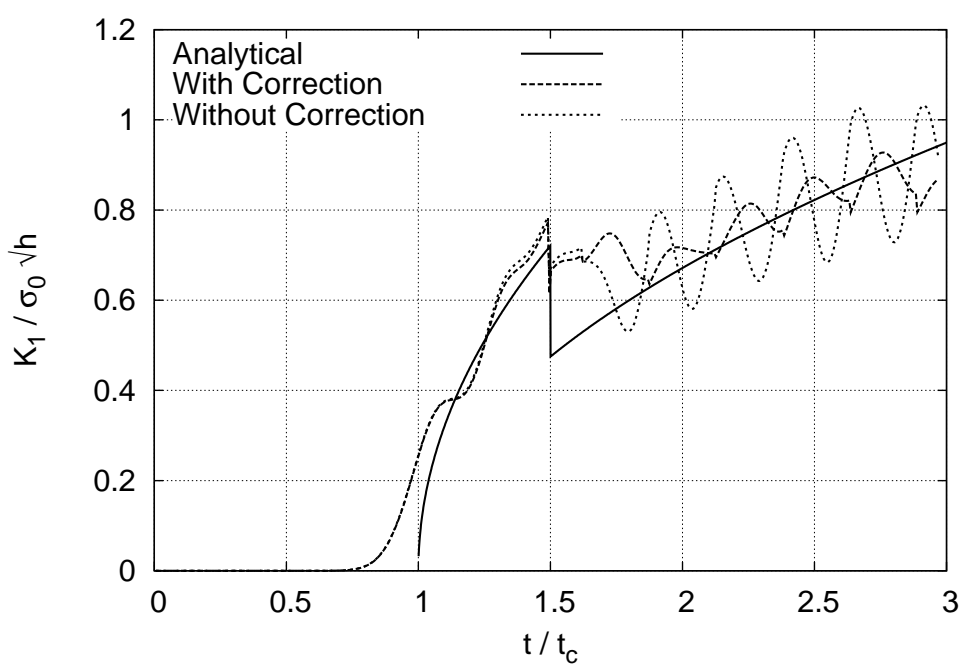

Figure 8. Normalized stress intensity factor as a function of time in the coarse mesh: analytical, numerical with and without correction force

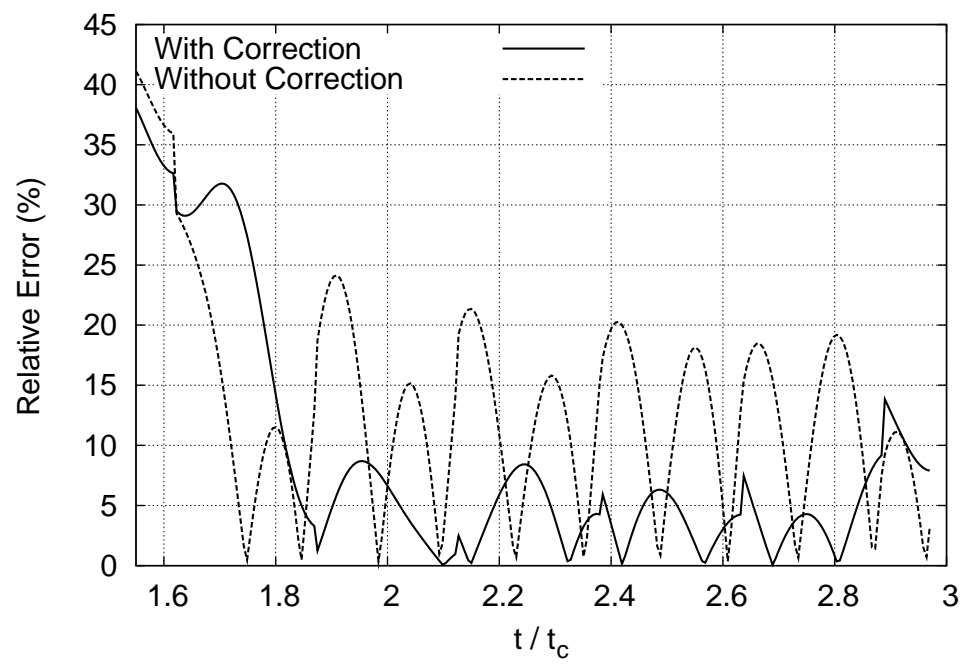

Figure 9. Relative error on stress intensity factor as a function of time in the coarse mesh with and without correction force 
478 European Journal of Computational Mechanics. Volume 18 - No. 5-6/2009

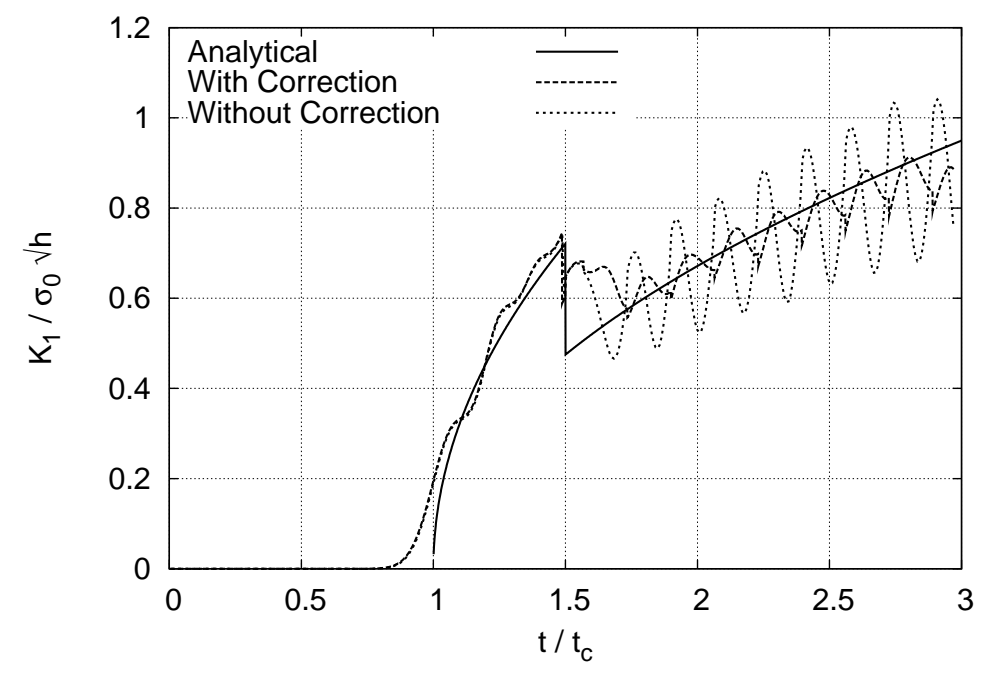

Figure 10. Normalized stress intensity factor as a function of time in the fine mesh: analytical, numerical with and without correction force

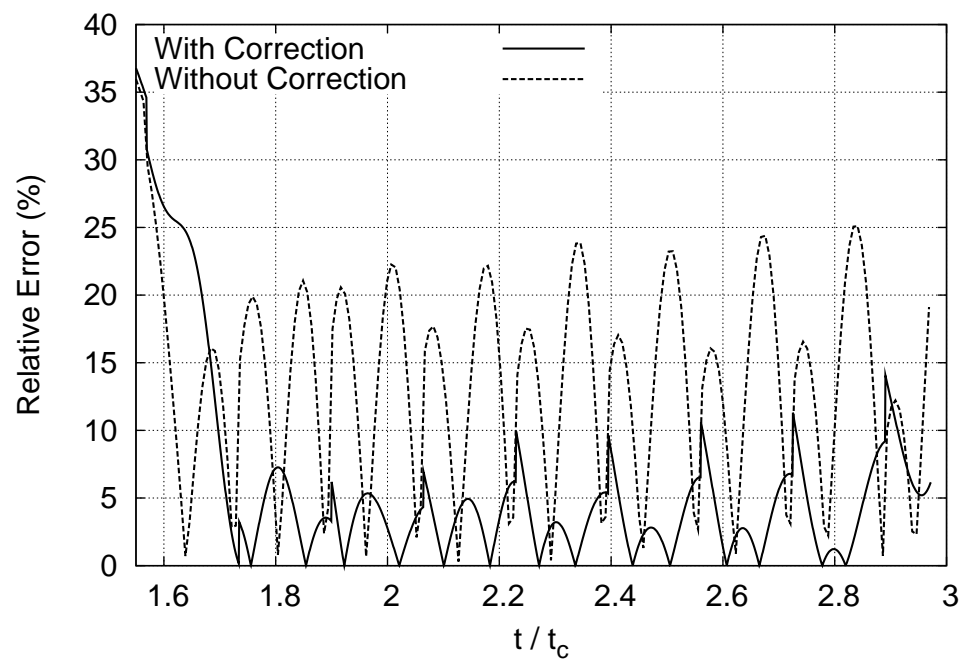

Figure 11. Relative error on stress intensity factor as a function of time in the fine mesh with and without correction force 

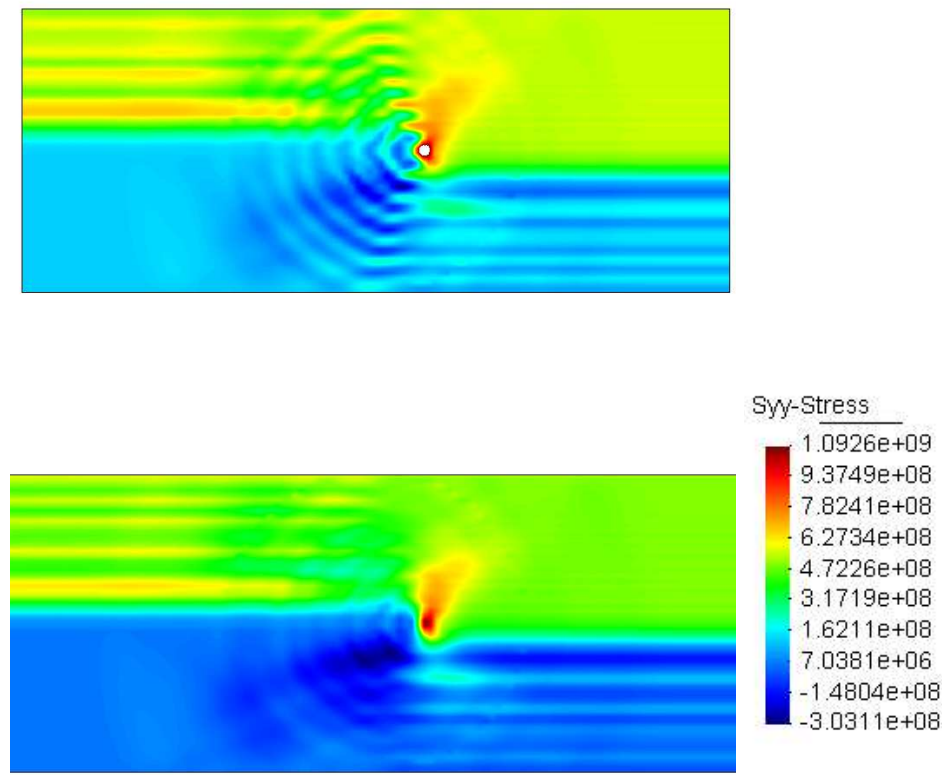

Figure 12. Stress field in the fine mesh: upper without correction and with correction at the bottom

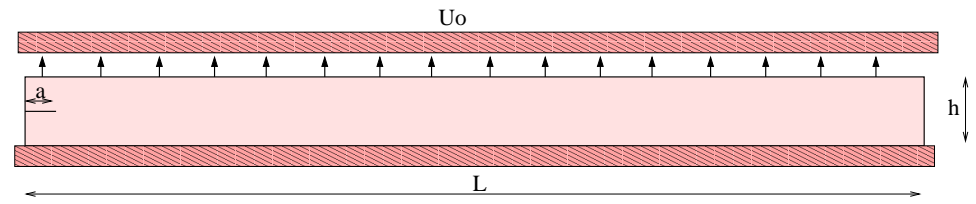

Figure 13. Geometry and loading

\subsection{Infinite strip in mode 1}

We consider a mode 1 crack propagation as shown in Figure 13. The bottom of the specimen is fixed, whereas the top is constrained to a vertical displacement. The initial crack is horizontal; thus a mode 1 crack propagation occurs. The dimensions of the specimen shown in Figure 13 are the following: length $L=2 \mathrm{~mm}$, height $h=0.2 \mathrm{~mm}$, and the initial crack length $a=0.09 \mathrm{~mm}$. The imposed displacement $U_{0}$ is equal to $0.007 \mathrm{~mm}$. The material model is linear elastic. The material properties are presented in Table 2. A cohesive law is used for the crack propagation; the fracture energy is $G_{f}=352.3 \mathrm{~Pa} . \mathrm{m}$. The specimen is discretized with 90 by 9 4-node elements. Two computations are run: with or without correction force. As expected, the crack path is strictly straight. 
Table 2. Material properties for the pure mode 1 test

\begin{tabular}{lcr}
\hline Material property & Symbol & Value \\
\hline Young's modulus & $E$ & $3.24 \mathrm{GPa}$ \\
Poisson's ratio & $\nu$ & 0.35 \\
Density & $\rho$ & $1,090 \mathrm{~kg} / \mathrm{m}^{3}$ \\
Cohesive law & $G_{F}$ & $352.3 \mathrm{~Pa} . \mathrm{m}$ \\
\hline
\end{tabular}

Figure 14 presents the crack lengths and the mode 1 stress intensity factors as a function of time for both computations: with or without correction force. One notices that both results are similar in time in term of stress intensity factors and crack length. Figure 15 presents the different energies (kinetic, deformation and fracture) as a function of time. Both simulations give similar results in term of energy, and only one curve is seen in Figure 15 instead of two. Moreover, Figure 15 presents the sum of all different energies as a function of time during the crack propagation. Both curves are similar, and show a constant total energy during the propagation. This result is important to underline the energy conservation in this simulation, even with the correction force.

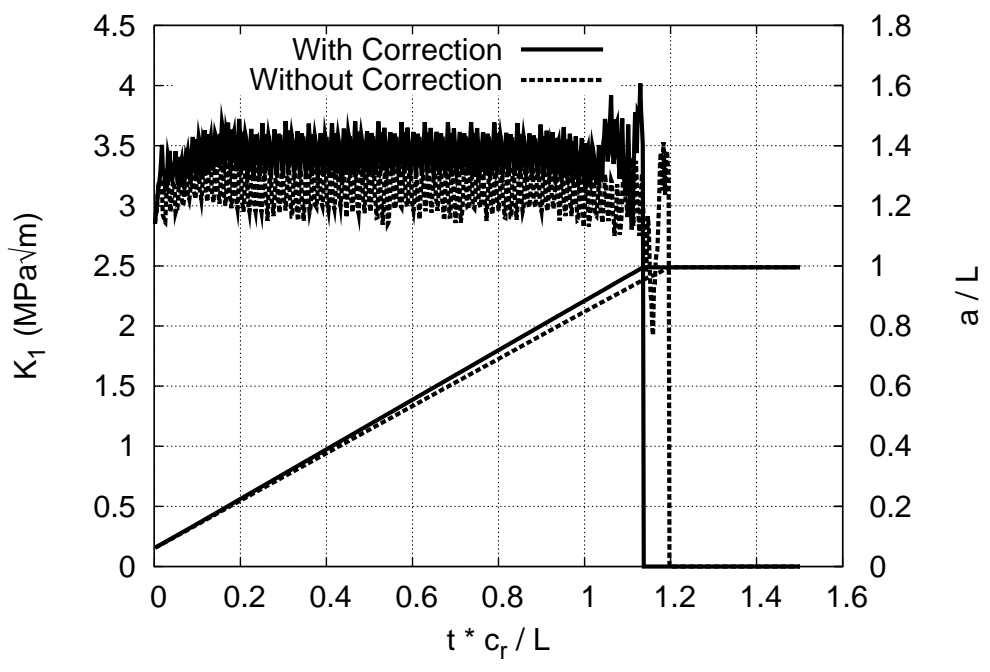

Figure 14. Crack lengths and mode 1 stress intensity factors as a function of time 


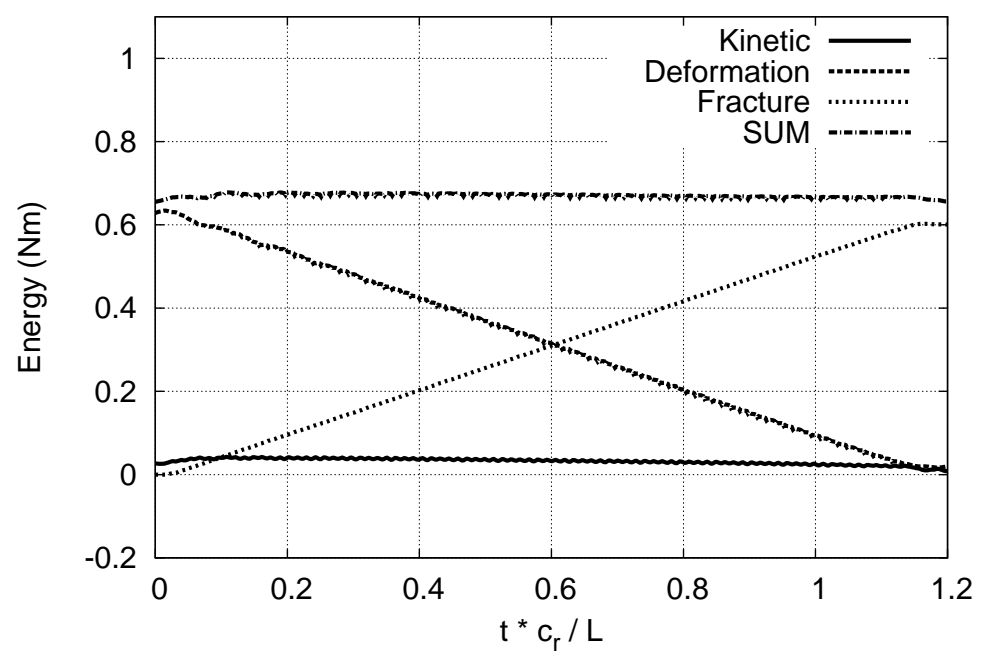

Figure 15. Energies as a function of time (Kinetic, Deformation and Fracture energies)

\section{Conclusion}

We have proposed a method to describe the release of the crack tip element in methods just a discontinuous enrichment in XFEM. This method is based on the continuity of the internal forces corresponding to the newly added degrees of freedom; indeed it makes the corresponding acceleration continuous, and thus velocity and displacement through the explicit Newmark scheme. The control of the acceleration is performed through the use of a correction force and the position of the virtual crack tip in the element. The restriction is that the crack has to be driven by a criterion related to the crack velocity; e.g. the stress intensity factors. However, we have shown that the use of this correction force improves the accuracy of the stress intensity factors during propagation because it significantly decreases the nonphysical stress oscillation near crack tip.

Acknowlegment

The authors gratefully acknowledge the support of the Army Research Office through Grant W911NF-08-1-0212 and the Office of Naval Research through Grant N00014-08-1-1191. 


\section{References}

Barenblatt G., " The mathematical theory of equilibrium cracks formed in brittle fracture", Advanced Applied Mechanics, vol. 7, p. 55-129, 1962.

Belytschko T., Liu W., Moran B., Nonlinear finite elements for continua and structures, Wiley, Chichester, 2000.

Belytschko T., Moës N., Usui S., Parimi C., “ Arbitrary discontinuities in finite elements”, International Journal for Numerical Methods in Engineering, vol. 50, n 4, p. 993-1013, 2001.

Benvenuti E., “ A regularized XFEM framework for embedded cohesive interfaces”, Computer Methods in Applied Mechanics and Engineering, vol. 197, $\mathrm{n}^{\circ}$ 49-50, p. 4367-4378, 2008.

Black T., Belytschko T., " Elastic crack growth in finite elements with minimal remeshing”, International Journal for Numerical Methods in Engineering, vol. 45, p. 601-620, 1999.

Bui H., Mecanique de la rupture fragile, Masson, 1978.

Combescure A., Gravouil A., Gregoire D., Réthoré J., “ X-FEM a good candidate for energy conservation in simulation of brittle dynamic crack propagation", Computer Methods in Applied Mechanics and Engineering, vol. 197, $\mathrm{n}^{\circ}$ 5, p. 309-318, 2008.

Dolbow J., Moës N., Belytschko T., “ An extended finite element method for modeling crack growth with frictional contact”, Computer Methods in Applied Mechanics and Engineering, vol. 190, p. 6825-6846, 2001.

Duan Q., Song J., Menouillard T., Belytschko T., “ Element-local level set method for threedimensional dynamic crack growth”, International Journal for Numerical Methods in Engineering, 2009.

Freund L., " Crack Propagation in an Elastic Solid Subjected to General Loading. Pt. 1. Constant Rate of Extension", Journal of the Mechanics of Physics and Solids, vol. 20, n 3, p. 129140, 1972.

Freund L., Dynamic Fracture Mechanics, Cambridge University Press, 1990.

Freund L., Douglas A., " Influence of inertia on elastic-plastic antiplane-shear crack growth.”, Journal of the Mechanics of Physics and Solids, vol. 30, n 1, p. 59-74, 1982.

Hansbo A., Hansbo P., " A finite element method for the simulation of strong and weak discontinuities in solid mechanics", Computer Methods in Applied Mechanics and Engineering, vol. 193, n 33-35, p. 3523-3540, 2004.

Hillerborg A., Modeer M., Petersson P. et al., “ Analysis of crack formation and crack growth in concrete by means of fracture mechanics and finite elements", Cement and Concrete Research, vol. 6, $\mathrm{n}^{\circ}$ 6, p. 773-782, 1976.

Khenous H., Laborde P., Renard Y., “ On the discretization of contact problems in elastodynamics", Lecture Notes in Applied and Computational Mechanics, vol. 27, p. 31-38, 2006.

Melenk J., Babuška I., “ The partition of unity finite element method: Basic theory and applications", Computer Methods in Applied Mechanics and Engineering, vol. 139, $\mathrm{n}^{\circ} 1-4$, p. 289-314, 1996.

Menouillard T., Elguedj T., Combescure A., “ Mixed-mode stress intensity factors for graded materials", International journal of solids and structures, vol. 43, n 7-8, p. 1946-1959, 2006. 
Menouillard T., Réthoré J., Combescure A., Bung H., “Efficient explicit time stepping for the eXtended Finite Element Method (X-FEM)”, International Journal for Numerical Methods in Engineering, vol. 68, p. 911-939, 2006.

Menouillard T., Réthoré J., Moës N., Combescure A., Bung H., “ Mass lumping strategies for X-FEM explicit dynamics: Application to crack propagation”, International Journal for Numerical Methods in Engineering, vol. 74, $\mathrm{n}^{\circ}$ 3, p. 447-474, 2008.

Menouillard T., Song J.-H., Duan Q., Belytschko T., “ Time Dependent Crack Tip Enrichment for Dynamic Crack Propagation”, International Journal of Fracture, 2009.

Moës N., Belytschko T., “ Extended finite element method for cohesive crack growth”, Engineering Fracture Mechanics, vol. 69, n 7, p. 813-833, 2002.

Moës N., Dolbow J., Belytschko T., “ A finite element method for crack growth without remeshing”, International Journal for Numerical Methods in Engineering, vol. 46, n 1, p. 131150, 1999.

Ortiz M., Pandolfi A., " Finite-deformation irreversible cohesive elements for three-dimensional crack-propagation analysis”, Int. J. Numer. Methods Eng, vol. 44, n 9, p. 1267-1282, 1999.

Patzák B., Jirasek M., “ Process zone resolution by extended finite elements”, Engineering Fracture Mechanics, vol. 70, n 7-8, p. 957-977, 2003.

Rabczuk T., Song J., Belytschko T., "Simulations of instability in dynamic fracture by the cracking particles method”, Engineering Fracture Mechanics, vol. 76, p. 730-741, 2009.

Ravi-Chandar K., Dynamic fracture, Elsevier Science, 2004.

Remmers J., Borst R., Needleman A., “ A cohesive segments method for the simulation of crack growth”, Computational Mechanics, vol. 31, n 1, p. 69-77, 2003.

Réthoré J., Gravouil A., Combescure A., “ An energy-conserving scheme for dynamic crack growth using the extended finite element method", International Journal for Numerical Methods in Engineering, vol. 63, p. 631-659, 2005.

Rosakis A., Freund L., " Optical Measurement of the Plastic Strain Concentration at a Crack Tip in a Ductile Steel Plate", Journal of Engineering Materials and Technology (Transactions of the ASME), vol. 104, $\mathrm{n}^{\circ}$ 2, p. 115-120, 1982.

Song J., Areias P., Belytschko T., " A method for dynamic crack and shear band propagation with phantom nodes", International Journal for Numerical Methods in Engineering, vol. 67, p. 868-893, 2006.

Stolarska M., Chopp D., Moës N., Belytschko T., “ Modelling crack growth by level sets in the extended finite element method”, International Journal for Numerical Methods in Engineering, vol. 51, p. 943-960, 2001.

Unger J., Eckardt S., Könke C., “ Modelling of cohesive crack growth in concrete structures with the extended finite element method", Computer Methods in Applied Mechanics and Engineering, vol. 196, n 41-44, p. 4087-4100, 2007.

Received: 17 June 2009 Accepted: $1^{\text {st }}$ October 2009 
